\title{
FIGO Stage IV
}

National Cancer Institute

\section{Source}

National Cancer Institute. FIGO Stage IV. NCI Thesaurus. Code C96261.

A FIGO stage term that applies to gynecologic cancers. For cervical cancer, it refers to cancer that invades the mucosa of bladder or rectum, and/or extends beyond the true pelvis (FIGO stage IVA), or to cancer with distant metastases (FIGO stage IVB); for endometrial cancer, it refers to cancer that invades the bladder mucosa and/or the bowel mucosa (FIGO stage IVA), or to cancer with distant metastases (FIGO stage IVB). 